\title{
DYSPHAGIA SCREENING PROTOCOL FOR ACUTE STROKE PATIENT: A LITERATURE REVIEW
}

\author{
MG Enny Mulyatsih ${ }^{1,2}$, Moses Glorino Rumambo Pandin ${ }^{3}$ \\ ${ }^{1}$ Faculty of Nursing, Airlangga University Surabaya \\ ${ }^{2}$ National Brain Center Hospital Prof. Dr.dr. Mahar Mardjono \\ ${ }^{3}$ Faculty of Humanities, Airlangga University Surabaya \\ Email: $\underline{\text { mg.enny.mulyatsih-2020@ fkp.unair.ac.id }}$
}

\begin{abstract}
Background: Nearly two-thirds of acute stroke patients have dysphagia. Dysphagia is difficulty to swallow food or liquids. Early detection of dysphagia is crucial in stroke patients as a result of increased morbidity and mortality due to malnutrition and respiratory tract infections.

Aim: Our purpose was to conduct a literature review of dysphagia screening for stroke patient.

Methods: We used the bolean operator to search articles of "or" and "and" with the key words were "Dysphagia" or "Screening", AND "Stroke" or Acute Stroke" AND "Nursing". Data based used were Scopus, Proquest and Science Direct with inclusion criteria using full text in English which published from 2019 to 2021 . We obtained 240 articles and then we screened by reading the main focus of articles with paying attention to the topics and the suitability of article content. Result: Twenty five publications relating to dysphagia screening met the inclusion criteria. There are five methods of dysphagia screening performed by nurses or other health workers: 1) a simple Questionnaire Test (4QT) method; 2) Water Swallow Test (WST) method; 3) Bed Side Screening Tool for Dysphagia (BSTD) method; 4) Volume Viscosity Swallow Test (V-VST) method; 5) EAT-10 method.

Conclusion: screening is the first step in the identification of swallowing impairment or dysphagia of stroke patient. Dysphagia is an independent predictor of poor patient outcome and prolonged recovery time. Nurse has an important role to conduct a screening and must ensure that the selected tools has high reliability and concurrent validity.
\end{abstract}

Key Words: Dysphagia, Nursing, Screening, Stroke 


\section{INTRODUCTION}

Stroke is a leading cause of disability and death worldwide (1). The priority of nursing intervention are to maintain nutrition and ensure that stoke patient could swallow safely without aspiration $(1,6)$. Stroke may lead to mild, moderate or severe swallowing difficulties. Several recent studies highlight the impact of dysphagia on dehydration, malnutrition, and aspiration pneumonia (6). Aspiration pneumonia was associated with prolonged hospital stay; worsening stroke patient outcome, and increased mortality (6).

Dysphagia defined as difficulty in food or liquid passing from the mouth, through the pharynx to the esophagus and onwards to the stomach (6). Approximately 65-90\% of dysphagia patients experience disturbances in the oropharyngeal phase, changes in lung function, risk of aspiration, nutrition, and quality of life (5). However, in some patients dysphagia will improve spontaneously within one week after the attack, about 50\% will persist for up to six months, and a small proportion will be permanent (7). A study conducted by Paiva et al showed that stroke patients with dysphagia and had nasogastric tube increased the risk of disability, almost 15 times than pasient non dysphagia (p 0,002 OR=14,97).

Dysphagia screening can detect swallowing problems early in acute stroke patients and prevent aspiration or other complications such as dehydration or nutritional problems (8). According to the AHA recommendations, screening for dysphagia should be performed before giving the stroke patient any food, water, or an oral medicine (9). Stroke patient with dysphagia to have more likely to develop pulmonary infection $(13,1 \%$ versus $1,9 \%)$, to have more difficulty in activity daily livings $(52,4 \%$ versus $18,0 \%)$, and to have prolonged length of stay at hospital $(14,0 \%$ versus $4,3 \%)$. The AHA also recommends that dysphagia screening be carried out by speech therapists or other trained health professionals (9). Patients can have a stroke at any time of the morning, afternoon, or evening. Because nurses are on duty 24 hours in the Emergency Room or the Stroke Unit, it is the nursing profession that is most likely to screen for dysphagia (1). A qualitative study on dysphagia screening by Couto and Oliveira showed that nurses had different perceptions and used different protocols and instruments to conduct dysphagia screening (1)

Dysphagia is associated with worse functional outcomes, which entails the poorer quality of life, and increased length of stay (1). Various screening methods for dysphagia have been developed and have satisfactory sensitivity and specificity. There is a need for protocol and 
dysphagia screening policies for stroke patients in the emergency room or the Stroke Unit by hospital management so that dysphagia screening can be carried out on time and procedures. Dysphagia screening in acute stroke must be administered by nurses as early as possible so that patients are not kept nil by mouth for unnecessary time.

\section{METHOD}

The design of this research is a literature review. The article should describe the variables corresponding to the problem discussed. Search for articles using the operator of "or" and "and". The data collection was carried out in April 2021 with the keywords "Dysphagia" and "Stroke" and "Nursing". Data based used were Scopus, Science Direct, and Proquest with inclusion criteria using full text, the document type is an article and English language in the period of 2019 to 2021. We obtained 240 articles which were 133 from Scopus, 10 from Science Direct, and 97 from Proquest. The articles were then screened by reading the main article part with focusing on the topic and the suitability of the article content from the abstract, methods, and the result. In the end, seven articles matched the predetermined criteria and were used in this literature review.

\section{RESULT}

Table 1 Distribution of current dysphagia screening methods and the result

\begin{tabular}{|c|c|c|}
\hline $\begin{array}{l}\text { Researcher, } \\
\text { Year }\end{array}$ & Title & Result \\
\hline $\begin{array}{l}\text { Oliveira et al, } \\
2020\end{array}$ & $\begin{array}{l}\text { Nurses' preferred items for } \\
\text { dysphagia screening in acute } \\
\text { stroke patients: A qualitative } \\
\text { study }\end{array}$ & $\begin{array}{l}20 \text { nurses have different perceptions based on } \\
\text { patient clinical data and the screening } \\
\text { instruments (1) }\end{array}$ \\
\hline $\begin{array}{l}\text { Eltringham et } \\
\text { al, } 2019\end{array}$ & $\begin{array}{l}\text { Variation in dysphagia } \\
\text { assessment and management in } \\
\text { acute stroke: An interview } \\
\text { study }\end{array}$ & $\begin{array}{l}\text { Fifteen nurses from five hospitals thought that } \\
\text { there was no standardized dysphagia screening } \\
\text { protocol in the hospital (10) }\end{array}$ \\
\hline $\begin{array}{l}\text { Tsang et al, } \\
2020\end{array}$ & $\begin{array}{l}\text { A New Simple Screening } \\
\text { Tool-4QT: Can It Identify } \\
\text { Those with Swallowing } \\
\text { Problems? A Pilot Study }\end{array}$ & $\begin{array}{l}100 \% \text { sensitivity, } 80.4 \% \text { specificity. The } 4 \mathrm{QT} \\
\text { instrument is very sensitive for screening for } \\
\text { dysphagia but is not specific for stroke } \\
\text { patients. A positive prediction of } 50 \% \text {, only } \\
50 \% \text { of patients screened for dysphagia after } \\
\text { reconfirmation by speech therapists that it is } \\
\text { true dysphagia (7). }\end{array}$ \\
\hline
\end{tabular}


A nurse applying the Simple Water Swallow test was subsequently reconfirmed by the Systematic dysphagia screening of elderly persons in the Melgaard et al, emergency department-a $\underline{2020}$ feasibility study occupational therapist. The result is that screening for dysphagia in the elderly can be carried out by nurses in the Emergency Room (3)

Nurses screened using BSTD in $33.3 \%$ of Diagnostic Accuracy of a cases and speech therapists in $30 \%$ of cases. Bedside Screening Tool for The result is Cohen K 0.92 (optimal suitability Immovilli, Dysphagia (BSTD) in Acute when K>0.8), sensitivity 100\%, specificity $\underline{2021}$ Stroke Patients $95.2 \%(8)$

The Modified Volume-

Viscosity Swallow Test as a The dysphagia screening method using the Predictor of Aspiration modified V-VST is an easy-to-use and reliable Dong, Huang, Pneumonia after Acute screening tool for the detection of dysphagia in \& Dong, 2021 Ischemic Stroke stroke patients (2)

Simplified cough test can The Simplicity Cough Test method is a strong Masahiro et al, predict the risk for pneumonia indicator for predicting pneumonia in acute 2020 in patients with acute stroke stroke patients (11) The V-VST is a validated method to screen the

Lesion location and other patient ability of swallowing. Nurses have to predictive factors of dysphagia and its complication in acute Ana et al, 2019 stroke consider the signs of impaired efficacy of swallow like as changes in voice quality (including wet voice), cough or a decrease in oxygen saturation

\section{DISCUSSION \\ DYSPHAGIA}

Stroke is the first leading cause of death in Indonesia. Most of the causes of death of stroke patients are lung infections as a complication of dysphagia (13), with a mortality rate of $12.8 \%$ higher than stroke patients without dysphagia (3). In general, there is about $8 \%$ of the population in the world has dysphagia, and the incidence of dysphagia in stroke patients is very high, reaching almost $80 \%(1,3,4,8)$. Dysphagia is a disorder in swallowing food and or fluids, as a result of muscle weakness in swallowing in stroke patient or as a result of the aging process. Nearly half of the elderly in Spain (47.4\%) who are over 70 years old experienced dysphagia (4). Dysphagia can lead to complications, such as aspiration pneumonia (2). A study in Taiwan found that dysphagia is a critical factor in aspiration pneumonia and malnutrition (14).; dehydration; and malnutrition, which in turn can lead to prolonged hospital stay (5); psychological disorders such as stress, anxiety, and depression; decreased quality of life (15); increased health costs; increased mortality 
and decreased patient outcomes after stroke (16). Based on a study by Takeda et al, aspiration was observed in 16 of 172 patient when swallowing $3 \mathrm{ml}$ of water during Video Fluoroscopy was conducted (17). Another important result from study by Meschi et al is the association between dysphagia and low physical performance (18). Many stroke patients recovery swallowing spontaneously, but 11\%-50\% still have dysphagia at 6 months (19). Post stroke dysphagia may cause aspiration of oral secretion, ingested food or liquids and to be the primary risk factor to pneumonia (19). Management of dysphagia patients is carried out by a multidisciplinary team approach, consisting of doctors, nurses, speech language pathologist, occupational therapists, and nutritionists (13). The nurse uses a nursing process that begins with an assessment, specifically the assessment of swallowing function by screening for dysphagia (4). The results of a study on nurses' perceptions regarding the assessment of swallowing function in stroke patients are very important, but the results of these assessments are not well documented, especially if they do not use protocol or instruments screening (14)

\section{DYSPHAGIA SCREENING}

The purpose of screening for dysphagia is to screen whether or not it is safe for patients to eat, drink and take oral medications (4). Screening is a means of judiciously selecting individuals who need a comprehensive examination. One key feature to the interpretation of screening approaches is that they are pass or fail procedures (20). According to the recommendations of the AHA, all stroke patients should be treated with dysphagia screening before eating, drinking, or receiving oral medication (9). Screening for dysphagia should be carried out as early as possible in the Emergency Room, so that safe feeding and drinking techniques can be established for patients to prevent aspiration, which is less than one hour after the patient is admitted to the Emergency Room (5). Based on the result of study by Fairfield and Smithard, the majority of dysphagia screening (93\%) was performed for less than 24 hours, and only $34 \%$ of them took less than 4 hours (4). The AHA recommends that screen for dysphagia in stroke patients to be performed by a speech language pathologist or other trained health care provider (9). Slightly different while the examinations of dysphagia using a special tools such as Video-fluoroscopy (VFS) or Fiberoptic Endoscopy Evaluation of Swallowing (FEES) must be done by a doctor, and the Carotis Auscultation should be done by a speech language pathologist (4). However, there is no consensus in four countries, namely the US, UK, Canada, and Australia, regarding who is most 
recommended for dysphagia screening in the Emergency Room. Nurses are essential in the emergency team and should ideally be able screen and priorities dysphagia management in stroke patient (21). The nursing profession that is more likely to screen for dysphagia, because nurses are on duty 24 hours in the Emergency Room or Stroke Unit (1). A cross sectional study by Knight et al showed the majority nurses $(n=100 ; 76,9 \%)$ had care for stroke patients with swallowing difficulty, with only $26,9 \%$ having received training about stroke care (22). Otherwise, based on study by Oliveira, Couto, and da Mota, the results of screening for dysphagia by nurses are as valid as those carried out by other professions (1). Likewise, the study results of Immovilli et al showed an Excellent Agreement $>0.8$ between screening performed by nurses and Speech Pathologist or Occupational Therapist (22)(1). Another study shows that Allied Health Assistance (AHAs) also demonstrated the ability to conduct dysphagia screening on a cohort study (23). On the other hand, a study from knight et al found that nurses across all levels had only moderate knowledge regarding identification and management of acute stroke (22).

\section{METHODES AND INSTRUMENT OF SCREENING}

There is no consensus on the method of screening for dysphagia between hospitals, so the use of protocols or instruments still varies (24). The oldest and most frequently used method is the Water Swallowing Test (WST) (6). The test by giving water varies depending on the perception of the nurse, ranging from $3 \mathrm{ml}$ to $60 \mathrm{ml}$. Although the sensitivity and reliability of WST are satisfactory, there is a risk that the patient aspirates and experiences oxygen desaturase, so pulse oximetry and oxygen must be prepared. The second instrument is a questionnaire containing 4 questions to the patient or family or 4QT (7). Questions include: is there a cough or choking after eating; does it take longer than usual to eat more; is there a change in consistency with meals; and whether there is a change in voice after eating or drinking. Although this method is very sensitive, it is less specific to use for fusing dysphagia in stroke patients (4). The next method is the Oropharyngeal Dysphagia Screening Test for Patients and Professionals (ODS-PP). This method is also a questionnaire for patients consisting of 18 question items, among others: is there a cough or choking during and after meal or drinking; is there difficulty of breath; whether there is a change in voice after eating or drinking. In general, the ODS-PP method has high reliability and concurrent validity (25). The next method is The Volume-Viscosity Swallow Test (V-VST) is a bedside method to screen patients for dysphagia (12). The V-VST was designed as an effort test to identify 
clinical signs of impaired efficacy (efficacy of labial seal, presence of oral or pharyngeal residue and presence of piecemeal deglutition) and safety (voice changes, cough and decrease in oxygen saturation $\geq 3 \%$ ) of swallow. To protect the safety of patients, the test starts with nectar viscosity and increasing bolus volumes (from 5 to 10 and $20 \mathrm{ml}$ ) in a progression of increasing difficulty. If patients complete the nectar series without cough or aspiration, a less safe liquid viscosity series is assessed and then a safer pudding viscosity series is performed in the same way. The study showed that the accuracy of the V-VST inpatients was safe, quick and accurate, with sensitivity and specificity of $88.2 \%$ and $64.7 \%$, respectively (12). The last method that we reviewed was the instrument EAT-10. The Ten 10-item Eating Assessment Tool (EAT-10) is a symptom-specific tool, commonly used in clinical practice. The questionary contain of 10 item, with a maximum total score of 40 points. Score is 3 or greater than 3 points suggests a potential swallowing problem (18). The ten questions including: 1) The swallowing problem make patient lose; 2) The swallowing problem interferes patient ability to go out for meals; 3) Patient takes extra effort to swallow liquids; 4) Patient takes extra effort to swallow solids; 5) Patient takes extra effort to swallow pills ; 6) Patient feel painful when swallow; 7 The patient pleasure of eating is affected by swallowing; 8) When patient swallow food sticks in their throat; 9) Patient cough when they eat; 10) Swallowing is stressful for patient (18).

\section{CONCLUSION}

Dysphagia in stroke patients must be managed properly, starting from early detection through dysphagia screening as part of nursing assessment. The objectives of screening are to prevent aspiration and establish efficient and safe oral intake for stroke patients. Screening should carried out as early as possible less than one hour after the patient arrives at the Emergency Room. The methods and instruments are used must be simple, require a short time, have a low risk, and the screening tool would have both high sensitivity and high specificity.

\section{REFERENCES}

1. Oliveira IDJ, Couto GR, Andreia L. Nurses' preferred items for dysphagia screening in acute stroke patients : A qualitative study Nursing Practice Today Nurses' preferred items for dysphagia screening in acute stroke patients : A qualitative study Stroke is a neurological deficit, attri. Nurs Pract Today. 2020;7(June):226-33.

2. Dong Y, Hu B, Huang S, Ye T, Dong Q. The Modified Volume-Viscosity Swallow Test as a Predictor of Aspiration Pneumonia after Acute Ischemic Stroke. Clin Neurol 
Neurosurg [Internet]. 2021;200:106351. Available from: https://doi.org/10.1016/j.clineuro.2020.106351

3. Melgaard D, Sørensen LR, Lund D, Leutscher P, Ludwig M. Systematic Dysphagia Screening of Elderly Persons in the Emergency Department — A Feasibility Study. Geriatrics. 2020;5:1-7.

4. Fairfield CA, Smithard DG. Assessment and Management of Dysphagia in Acute Stroke : An Initial Service Review of International Practice. Geriatrics. 2020;5:1-13.

5. Paiva R De, Thomaz J, Watson P, Bazan Z, Pinheiro G, Polegato BF, et al. Dysphagia and tube feeding after stroke are associated with poorer functional and mortality outcomes. Clin Nutr. 2020;30:2786-92.

6. Wright DJ, Smithard DG. Optimising Medicines Administration for Patients with Dysphagia in Hospital : Medical or Nursing Responsibility? Geriatrics. 2020;5:1-10.

7. Tsang K, Lau ESY, Shazra M, Eyres R, Hansjee D, Smithard DG. A New Simple Screening Tool - 4QT : Can It Identify Those with Swallowing Problems? A Pilot Study. Geriatrics. 2020;5:1-9.

8. Immovilli P, Rota E, Morelli N, Marchesi E, Terracciano C, Zaino D, et al. Diagnostic Accuracy of a Bedside Screening Tool for Dysphagia (BSTD ) in Acute Stroke Patients. J Stroke Cerebrovasc Dis [Internet]. 2021;30(2):105470. Available from: https://doi.org/10.1016/j.jstrokecerebrovasdis.2020.105470

9. Powers WJ, Rabinstein AA, Ackerson T, Adeoye OM, Bambakidis NC, Becker K, et al. AHA / ASA Guideline Guidelines for the Early Management of Patients With Acute Ischemic Stroke : 2019 Update to the 2018 Guidelines for the Early Management of Acute Ischemic Stroke. 2019.

10. Eltringham SA, Smith CJ, Pownall S, Sage K. Variation in Dysphagia Assessment and Management in Acute Stroke: An Interview Study. Geriatrics. 2019;4:1-12.

11. Id MN, Imamura E, Kuwabara M, Ayukawa T, Tachiyama K, Kamimura T, et al. Simplified cough test can predict the risk for pneumonia in patients with acute stroke. PLoS One [Internet]. 2020;1-12. Available from: http://dx.doi.org/10.1371/journal.pone.0239590

12. Cant A, Santamaría-nieto A, Díaz-ortega C, Rodríguez-y M, Angel M. Clinical Nutrition ESPEN Lesion location and other predictive factors of dysphagia and its complications in acute stroke n. 2019;33:178-82.

13. Wang T, Zhao Y, Guo A. International Journal of Nursing Sciences Association of swallowing problems with frailty in Chinese hospitalized older patients. Int J Nurs Sci [Internet]. 2020;7(4):408-12. Available from: https://doi.org/10.1016/j.ijnss.2020.09.005

14. Feng M, Lin Y, Chang Y, Chen C, Chiang H, Huang L, et al. The Mortality and the Risk of Aspiration Pneumonia Related with Dysphagia in Stroke Patients. J Stroke Cerebrovasc Dis [Internet]. 2019;28(5):1381-7. Available from: https://doi.org/10.1016/j.jstrokecerebrovasdis.2019.02.011

15. Rönnefarth M, Hanisch N, Brandt AU, Mähler A, Endres M, Paul F, et al. Dysphagia Affecting Quality of Life in Cerebellar Ataxia - a Large Survey. The Cerebellum. 2020;19:437-45.

16. Guidelines CN. Chinese expert consensus on food and nutrition management for dysphagia ( 2019 version ). Asia Pac J Clin Nutr. 2020;29(March):434-44.

17. Takeda C, Yoshida M, Nakamori M, Hosomi N, Nagasaki T, Yoshikawa M, et al. Delayed Swallowing Re fl ex is Overlooked in Swallowing Screening Among Acute 
Stroke Patients. J Stroke Cerebrovasc Dis [Internet]. 2020;29(12):105303. Available from: https://doi.org/10.1016/j.jstrokecerebrovasdis.2020.105303

18. Meschi T, Maggio M. The risk of dysphagia is associated with malnutrition and poor functional outcomes in a large population of outpatient older individuals. 2019;38:2684-9.

19. Juan W, Zhen H, Yan-ying F, Hui-xian Y, Tao Z, Pei-fen G, et al. A Comparative Study of Two Tube Feeding Methods in Patients with Dysphagia After Stroke: A Randomized Controlled Trial. J Stroke Cerebrovasc Dis [Internet]. 2020;29(3):104602. Available from: https://doi.org/10.1016/j.jstrokecerebrovasdis.2019.104602

20. Focus C. Swallowing Screening: Purposefully Different From an Assessment Sensitivity and Specificity Related to Clinical Yield, Interprofessional Roles, and Patient Selection. Am J Speech-Language Pathol. 2020;29(July):979-92.

21. Oliveira IDJ, Isabel S, Almeida F De, Couto GR. Conceptualization of nursing care to the person with post-stroke dysphagia Research question. Rev Enferm Ref. 2020;5:1-7.

22. Africa S, Africa S. Nurses ' knowledge of stroke-related oropharyngeal dysphagia in the Eastern Cape, South Africa. South African J Commun Disord. 2020;1-8.

23. Research ArticleExploring the Validity and Operational Impact of Using Allied Health Assistants to Conduct Dysphagia Screening for Low-Risk Patients Within the Acute Hospital Setting. Am J Speech Lang Pathol. 2020;29(November):1944-56.

24. Fortes AA, Westmark S. Primary Healthcare Professionals Experience of Transfer and Meaning According to Screening for Dysphagia. Geriatrics. 2019;4.

25. Quirós S, Serrano F, Mata S. Design and Validation of the Oropharyngeal Dysphagia Screening Test for Patients and Professionals : A Preliminary Study. Dysphagia [Internet]. 2020;35(1):52-65. Available from: https://doi.org/10.1007/s00455-019-09999-4 